\title{
Computed Tomography Findings for Intracerebral Hemorrhage Have Little Incremental Impact on Post-Stroke Mortality Prediction Model Performance
}

\author{
Darin B. Zahuranec $^{a}$ Brisa N. Sánchez ${ }^{b}$ Devin L. Brown ${ }^{a}$ \\ Jeffrey J. Wing ${ }^{b, c}$ Melinda A. Smith ${ }^{a}$ Nelda M. Garcia ${ }^{a}$ William J. Meurer ${ }^{a}$ \\ Lewis B. Morgenstern ${ }^{a, c}$ Lynda D. Lisabeth ${ }^{a, c}$ \\ a Stroke Program, University of Michigan Health System, Departments of ${ }^{b}$ Biostatistics and 'Epidemiology, \\ University of Michigan School of Public Health, Ann Arbor, Mich., USA
}

\section{Key Words}

Epidemiology $\cdot$ Cerebral infarction $\cdot$ Prediction of

outcome $\cdot$ Intracerebral hemorrhage

\begin{abstract}
Background: Stroke outcome studies often combine cases of intracerebral hemorrhage (ICH) and ischemic stroke (IS). These studies of mixed stroke typically ignore computed tomography (CT) findings for ICH cases, though the impact of omitting these traditional predictors of $\mathrm{ICH}$ mortality is unknown. We investigated the incremental impact of ICH CT findings on mortality prediction model performance. Methods: Cases of ICH and IS (2000-2003) were identified from the Brain Attack Surveillance in Corpus Christi (BASIC) project. Base models predicting 30-day mortality included demographics, stroke type, and clinical findings (National Institutes of Health Stroke Scale (NIHSS) +/- Glasgow Coma Scale (GCS)). The impact of adding CT data (volume, intraventricular hemorrhage, infratentorial location) was assessed with the area under the curve (AUC), unweighted sum of squared residuals $(\hat{S})$, and integrated discrimination improvement (IDI). The model assessment was performed first for the mixed case of $\mathrm{IS}$ and $\mathrm{ICH}$, and then repeated for $\mathrm{ICH}$ cases alone to determine whether any lack of improvement in
\end{abstract}

model performance with $\mathrm{CT}$ data for mixed stroke type was due to IS cases naturally forming a larger proportion of the total sample than ICH. Results: A total of 1,256 cases were included ( $86 \%$ IS, $14 \%$ ICH). Thirty-day mortality was $16 \%$ overall (11\% for IS; $43 \%$ for ICH). When both clinical scales (NIHSS and GCS) were included, none of the model performance measures showed improvement with the addition of $\mathrm{CT}$ findings whether considering IS and ICH together ( $\triangle \mathrm{AUC}$ : 0.002, 95\% Cl -0.01, 0.02; $\Delta \hat{\mathrm{S}}:-3.0,95 \% \mathrm{Cl}-9.1$, 2.6; IDI: 0.017, $95 \% \mathrm{Cl}-0.004,0.05)$ or considering ICH cases alone ( $\triangle \mathrm{AUC}$ : $0.02,95 \% \mathrm{Cl}-0.02,0.08 ; \Delta \hat{\mathrm{S}}:-2.0,95 \% \mathrm{Cl}-9.7,3.4$; IDI 0.065 , $95 \% \mathrm{Cl}-0.03,0.21)$. If NIHSS was the only clinical scale included, there was still no improvement in AUC or S when CT findings were added for the sample with $\mathrm{IS} / \mathrm{ICH}$ combined ( $\triangle$ AUC: $0.005,95 \% \mathrm{Cl}-0.01,0.02 ; \Delta \hat{\mathrm{S}}:-5.0,95 \% \mathrm{Cl}-11.6,1.0$ ) or for ICH cases alone ( $\Delta$ AUC: $0.05,95 \% \mathrm{Cl}-0.002,0.11 ; \Delta \hat{\mathrm{S}}$ : $-4.2,95 \% \mathrm{Cl}-11.5,2.3)$. However, IDI was improved when NIHSS was the only clinical scale for IS/ICH combined (IDI: $0.029,95 \% \mathrm{Cl} 0.002,0.065)$ and ICH alone (IDI: $0.12,95 \% \mathrm{Cl}$ $0.005,0.26)$. Conclusions: Excluding ICH CT findings had only minimal impact on mortality prediction model performance whether examining $\mathrm{ICH}$ and IS together or ICH alone. These findings have important implications for the design of clinical studies involving ICH patients.

Copyright $\odot 2012$ S. Karger AG, Basel

\section{KARGER}

Fax +41613061234 E-Mail karger@karger.ch www.karger.com

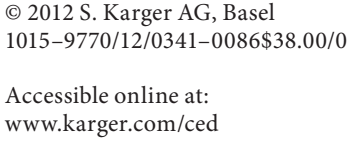

Darin B. Zahuranec, MD

University of Michigan Cardiovascular Center

1500 East Medical Center Drive, SPC \#5855

Ann Arbor, MI 48109-5855 (USA)

Tel. +1 734936 9075, E-Mail zdarin@ umich.edu 


\section{Introduction}

Stroke outcome studies often combine cases of intracerebral hemorrhage (ICH) and ischemic stroke (IS) to increase sample size. However, accounting for mortality risk in these studies of mixed stroke type is potentially problematic. Computed tomography (CT) findings such as hemorrhage volume, location, and intraventricular blood have been widely reported as powerful predictors of mortality in ICH [1-3], though these findings have no direct correlate in cases of IS. Mortality risk estimates for an individual may therefore be inaccurate if CT data are ignored for ICH.

Many large-scale population-based or quality improvement studies, such as those based on the European Registers of Stroke [4] or American Heart Association Get with the Guidelines database [5] do not traditionally report data on $\mathrm{ICH}$ volume. These and other studies investigating mixed populations of IS and ICH have either reported results stratified by stroke type, used a single baseline severity scale such as the National Institutes of Health Stroke Scale (NIHSS) across stroke types, or simply adjusted for stroke type as hemorrhagic or ischemic in regression models [4-9]. It is unknown if methods of severity adjustment which ignore $\mathrm{CT}$ data in $\mathrm{ICH}$ are adequate. While CT scans are readily available in clinical settings, obtaining original CT images to code detailed findings such as ICH volume and location for research studies adds extra expense and effort, particularly for large multicenter studies.

The primary goal of this study was to determine the incremental value of adding CT findings for ICH cases to models predicting 30-day mortality in a populationbased study combining ICH and IS. We compared the performances (discrimination and calibration) of models that contained demographics, exam findings, and stroke type to those that also incorporated CT data.

\section{Methods}

\section{Case Identification}

Data for this project came from the Brain Attack Surveillance in Corpus Christi (BASIC) project [10]. BASIC is a populationbased stroke surveillance project in Nueces County, Tex., USA. Detailed methods have been previously described [10-12]. Briefly, active and passive surveillance are used to identify all cases of stroke in the county. Board certified neurologists validate all cases of stroke and determine stroke type. IS is defined as the acute onset of a focal neurological deficit attributable to a specific cerebrovascular distribution that persisted for greater than $24 \mathrm{~h}$. ICH is defined as non-traumatic acute onset of focal neurological symptoms associated with a focal collection of blood within the brain parenchyma, excluding cases of hemorrhagic conversion of a cerebral infarction or tumor. The study population consisted of all BASIC patients with first-ever IS or ICH from January 1, 2000 through December 31, 2003. The time frame was restricted to these cases as ICH imaging data were available for this time period.

\section{Data Collection and ICH Scan Review}

Trained abstractors reviewed medical charts for key data. Initial NIHSS was abstracted from the chart by trained abstractors using a previously validated method [13] (97.5\%) or obtained from the medical record (2.5\%). Initial Glasgow Coma Scale (GCS) score was similarly obtained from the medical record $(54.1 \%)$ or calculated based on information abstracted from the medical record (45.9\%). Ethnicity was determined from the medical record and reported as either Mexican American or non-Hispanic white. Other ethnic groups were excluded from the analysis due to low numbers. CT scans of ICH patients were reviewed by study neurologists and coded for ICH volume, location as infratentorial or supratentorial, and presence or absence of intraventricular hemorrhage as previously described [14, 15]. These CT parameters were selected due to their known association with 30-day mortality [3]. ICH volume was calculated with the $\mathrm{ABC} / 2$ method [16]. Vital status at 30 days was determined from medical record review along with state and national databases as previously described [17]. Brain imaging studies were not available for detailed review for IS cases.

\section{Statistical Methods}

Baseline characteristics were compared by stroke type and by 30 -day mortality status using $t$ tests, $\chi^{2}$ tests, or non-parametric rank sum tests. We developed multivariable logistic regression models predicting 30-day mortality with and without the following CT findings: ICH volume (continuous), intraventricular hemorrhage (yes/no), and infratentorial hemorrhage (yes/no). Because CT findings are not available for IS cases, they were included only with their interaction with the stroke type indicator variable denoting that the stroke case was ICH. The models were: (1) the base model which included demographic information (age, gender, ethnicity), NIHSS, stroke type (IS $=0$ or ICH $=1$ ), and the interaction between NIHSS and stroke type; (2) the base model with the addition of ICH CT parameters; (3) the base model with GCS (modeled as a quadratic term due to non-linear association with log-odds of mortality) and the interaction between GCS and stroke type as additional severity measures, and (4) model 3 with ICH CT parameters. We assessed models with NIHSS only or NIHSS and GCS, since GCS is not routinely assessed in IS and may be unavailable in some datasets.

Predictive accuracy of models with and without CT findings was compared with three different metrics. The area under the receiver operating characteristic curve (AUC) $[18,19]$ and the integrated discrimination improvement (IDI) [20] were used to assess model discrimination, while model calibration was assessed with the unweighted sum of squared residuals $(\hat{S})$ [21]. Inference regarding improvement of models with CT findings was conducted by estimating the change in AUC and $\hat{S}$ ( $\triangle$ AUC and $\Delta \hat{S})$, the IDI index, and their 95\% CI. Models were considered to be significantly different from a comparison model if the 95\% CIs did not include zero. Bootstrapping [22] was used to construct CIs and to correct for over-fitting (i.e. deriving the model and as- 
Table 1. Description of the population by 30 -day mortality status and stroke type

\begin{tabular}{|c|c|c|c|c|c|c|c|}
\hline \multirow[t]{2}{*}{ Characteristic } & \multirow{2}{*}{$\begin{array}{l}\text { All patients } \\
(\mathrm{n}=1,256)\end{array}$} & \multicolumn{3}{|c|}{ 30-day mortality status } & \multicolumn{3}{|l|}{ Stroke type } \\
\hline & & $\begin{array}{l}\text { deceased at } \\
30 \text { days } \\
(n=197)\end{array}$ & $\begin{array}{l}\text { alive at } \\
30 \text { days } \\
(\mathrm{n}=1,059)\end{array}$ & $\mathrm{p}$ & $\begin{array}{l}\text { IS } \\
(n=1,085)\end{array}$ & $\begin{array}{l}\mathrm{ICH} \\
(\mathrm{n}=171)\end{array}$ & $\mathrm{p}$ \\
\hline Mean age (SD) & $71.1(12.5)$ & $75.9(13.1)$ & $70.2(12.2)$ & $<0.001$ & $70.9(12.5)$ & $72.2(12.9)$ & 0.23 \\
\hline Female sex & $655(52)$ & $122(62)$ & $533(50)$ & 0.003 & $566(52)$ & $89(52)$ & 0.98 \\
\hline Mexican American & $670(53)$ & $81(41)$ & $589(56)$ & $<0.001$ & $572(53)$ & $98(57)$ & 0.26 \\
\hline Hypertension & $860(68)$ & $129(65)$ & $731(69)$ & 0.33 & $741(68)$ & $119(70)$ & 0.73 \\
\hline Diabetes & $452(36)$ & $60(30)$ & $292(37)$ & 0.08 & $403(37)$ & $49(29)$ & 0.03 \\
\hline Coronary artery disease & $364(29)$ & 77 (39) & $287(27)$ & $<0.001$ & $320(30)$ & $44(26)$ & 0.31 \\
\hline Hyperlipidemia & $243(19)$ & $30(15)$ & $213(20)$ & 0.11 & $219(20)$ & $24(14)$ & 0.06 \\
\hline Atrial fibrillation & $131(10)$ & $39(20)$ & $92(9)$ & $<0.001$ & $117(11)$ & $14(8)$ & 0.3 \\
\hline Median NIHSS (IQR) & $4(2,8)$ & $14(6,26)$ & $3(1,6)$ & $<0.0001$ & $3(1,7)$ & $10(3,21)$ & $<0.0001$ \\
\hline Median GCS (IQR) & $15(14,15)$ & $10(6,14)$ & $15(14,15)$ & $<0.0001$ & $15(14,15)$ & $12(6,15)$ & $<0.0001$ \\
\hline 30-day mortality & $197(16)$ & - & - & & $123(11)$ & $74(43)$ & $<0.001$ \\
\hline \multicolumn{8}{|c|}{ CT parameters for ICH patients only $(n=171)$} \\
\hline Median ICH volume in cc (IQR) & $11(3,32)$ & $27(9,68)$ & $6(1,15)$ & $<0.0001$ & - & & - \\
\hline Intraventricular hemorrhage present & $90(53)$ & $56(76)$ & $34(35)$ & $<0.0001$ & - & & - \\
\hline Infratentorial hemorrhage & $21(12)$ & $14(19)$ & $7(7)$ & 0.02 & - & & - \\
\hline
\end{tabular}

Figures are n (\%) unless otherwise specified.

sessing predictive ability of the model on the same sample) [19]. Since ICH forms a naturally smaller subset of the total population, it is possible that lack of improvement in the model performance may be due to the fact that CT findings only improve the model fit for the minority of cases. To investigate this possibility, the assessment of model performance was repeated for ICH cases alone.

Statistical analysis was done in R version 2.9.2 and SAS version 9.2 (SAS Institute, Cary, N.C., USA). This project was approved by the Institutional Review Board of the University of Michigan and the individual Corpus Christi hospital systems.

\section{Results}

A total of 1,256 cases were included (IS 1,083 (86\%), ICH $171(14 \%))$. Descriptive characteristics of the population overall, by 30-day mortality, and by stroke type are shown in table 1. Most patients (IS 68\%, ICH 74\%) arrived to medical attention within 24 h of symptom onset. Compared to those with IS, patients with ICH were less likely to have diabetes, had a higher baseline NIHSS, and had a lower baseline GCS. Thirty-day mortality was 197/1,256 (16\%) overall, 123/1,083 (11\%) for IS, and 74/171 (43\%) for $\mathrm{ICH}$.
Parameter estimates for the multivariable logistic regression models predicting 30-day mortality are shown in online supplementary table E1 (for all online suppl. material, see www.karger.com/doi/10.1159/000339684). Table 2 demonstrates the fit parameters (AUC and $\hat{S}$ ) and measures of improvement ( $\Delta$ AUC, $\Delta \hat{S}$, and IDI) for these models. For the baseline model (model 1), which incorporated age, gender, ethnicity, NIHSS, stroke type, and the interaction between stroke type and NIHSS, the AUC was $0.83(95 \%$ CI $0.78,0.87)$ and $\hat{S}$ was 113.3 (95\% CI 93.3, 135.0). Adding the ICH CT findings (model 2 ) resulted in no significant change in AUC ( $\triangle$ AUC for model 2-model 1: $0.005,95 \% \mathrm{CI}-0.01,0.02)$, and slight but non-significant improvement in $\hat{S}(\Delta \hat{S}$ for model 2-model 1: -5.0 , 95\% CI -11.6, 1.0). However, the IDI comparing model 2 to model 1 was significantly different from zero, 0.029 (95\% CI 0.002, 0.065). The change in AUC and the IDI comparing model 2 and model 1 are shown in figure 1a, b. The area between the two receiver operating characteristic (ROC) curves (fig. 1a) is the $\triangle \mathrm{AUC}$, and the area between the sensitivity versus cutoff value curves (fig. 1b) is the IDI.

We also investigated a model that incorporated both NIHSS and GCS as measures of clinical severity (model 3). Adding CT data to model 3 did not result in any change in 
Fig. 1. Model comparison curves for $\mathrm{ICH}$ and IS combined. The comparison of model 1 (demographics, NIHSS, and stroke type $\times$ NIHSS) to model 2 (adding CT findings) is shown in a: ROC curve demonstrating the change in AUC, and $\mathbf{b}$ sensitivity versus probability cutoff curve demonstrating the IDI. The comparison of model 3 (model 1 plus GCS and GCS $\times$ stroke type) to model 4 (adding CT findings) is shown in c: ROC curve demonstrating the change in AUC, and d sensitivity versus probability cutoff curve demonstrating the IDI. Note that $\mathbf{b}$ demonstrates improvement in the sensitivity at larger probability cutoffs that leads to a positive and significant IDI. However, when GCS is added (d), the gain in sensitivity at large probability cutoffs is attenuated such that IDI is no longer significant.

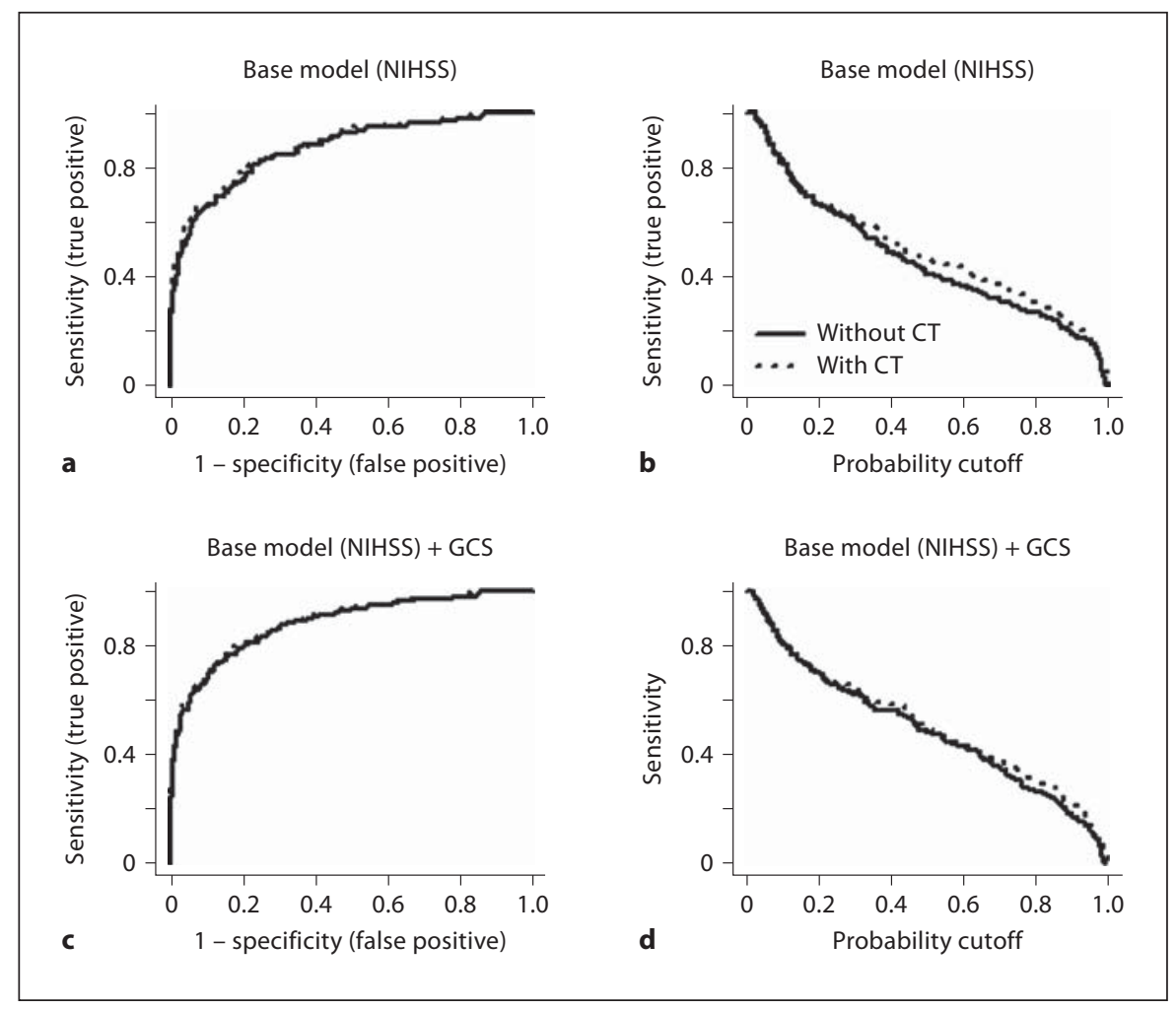

Table 2. Assessment of model performance: IS and ICH cases combined

\begin{tabular}{|c|c|c|c|c|c|c|c|}
\hline \multirow[t]{2}{*}{ Model } & \multirow[t]{2}{*}{ Covariates } & \multicolumn{2}{|c|}{$\begin{array}{l}\text { AUC (higher is better } \\
\text { discrimination) }\end{array}$} & \multicolumn{2}{|c|}{$\begin{array}{l}\hat{\mathrm{S}} \text { (lower is better } \\
\text { calibration) }\end{array}$} & \multicolumn{2}{|c|}{$\begin{array}{l}\text { IDI ( }>0 \text { is better } \\
\text { discrimination) }\end{array}$} \\
\hline & & value & $95 \% \mathrm{CI}$ & value & $95 \% \mathrm{CI}$ & value & $95 \%$ CI \\
\hline 1 & $\begin{array}{l}\text { Base model: age, gender, ethnicity, } \\
\text { NIHSS, stroke type, NIHSS } \times \text { stroke type }\end{array}$ & 0.83 & $0.78,0.87$ & 113.0 & $93.3,135.0$ & & \\
\hline 2 & Base model plus ICH CT findings* & 0.83 & $0.78,0.88$ & 108.0 & $87.6,130.0$ & & \\
\hline \multicolumn{2}{|c|}{ Change in AUC/S, or IDI (model 2-model 1) } & 0.005 & $-0.01,0.02$ & -5.0 & $-11.6,1.0$ & 0.029 & $-0.002,0.065$ \\
\hline 3 & Base model plus $\mathrm{GCS}^{\dagger}$, GCS $\times$ stroke type & 0.84 & $0.79,0.89$ & 109.0 & $89.4,129.0$ & & \\
\hline 4 & Model 3 plus ICH CT findings* & 0.85 & $0.80,0.89$ & 106.0 & $86.1,126.0$ & & \\
\hline \multicolumn{2}{|c|}{ Change in AUC/Ŝ, or IDI (model 4-model 3) } & 0.002 & $-0.01,0.02$ & -3.0 & $-9.1,2.6$ & 0.017 & $-0.004,0.051$ \\
\hline
\end{tabular}

* CT findings for ICH were included as interaction terms between stroke type and each of the following: (1) hemorrhage volume in cc, (2) intraventricular hemorrhage (yes/no), and (3) infratentorial hemorrhage (yes/no).

$\dagger$ Modeled as a quadratic term due to non-linear association with log-odds of mortality.

AUC ( $\Delta$ AUC for model 4-model 3: $0.002,95 \%$ CI -0.01 , $0.02)$ or $\hat{S}(\Delta \hat{S}$ for model 4-model 3: -3.0, 95\% CI -9.1, 2.6), or improvement in the IDI (IDI for model 4-model 3: 0.017, $95 \%$ CI $-0.004,0.05)$. The change in AUC and the IDI comparing model 4 and model 3 are shown in figure $1 \mathrm{c}, \mathrm{d}$.
The analysis was then repeated restricted to ICH cases alone, with model parameter estimates shown in online suppl. table E2 and measures of improvement shown in table 3. Increasing ICH volume and infratentorial location of hemorrhage was associated with risk of death 
Fig. 2. Model comparison curves for ICH cases only. The comparison of model 1 (demographics and NIHSS) to model 2 (adding CT findings) is shown in a: ROC curve demonstrating the change in AUC, and b sensitivity versus probability cutoff curve demonstrating the IDI. The comparison of model 3 (model 1 plus GCS) to model 4 (adding CT findings) is shown in c: ROC curve demonstrating the change in AUC, and d sensitivity versus probability cutoff curve demonstrating the IDI. Note that $\mathbf{b}$ and $\mathbf{d}$ demonstrate that sensitivity is actually decreased at small probability cutoffs when including CT findings, though the net IDI is positive and significant in $\mathbf{b}$. When GCS is added to the model (d), the gain in sensitivity at larger probability cutoffs is attenuated, resulting in a non-significant IDI.

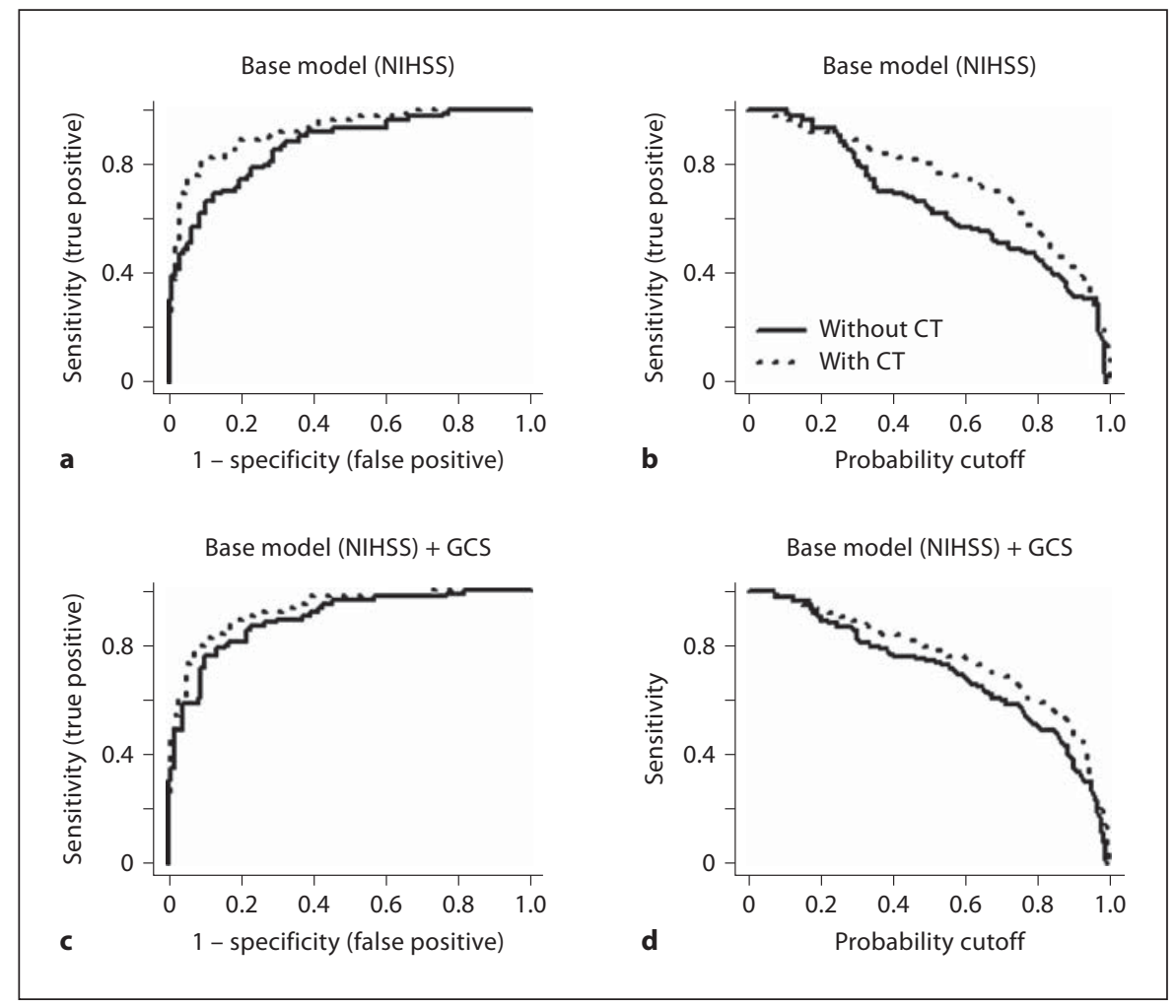

Table 3. Assessment of model performance: ICH cases only

\begin{tabular}{|c|c|c|c|c|c|c|c|}
\hline \multirow[t]{2}{*}{ Model } & \multirow[t]{2}{*}{ Covariates } & \multicolumn{2}{|c|}{$\begin{array}{l}\text { AUC (higher is better } \\
\text { discrimination) }\end{array}$} & \multicolumn{2}{|c|}{$\begin{array}{l}\hat{\mathrm{S}} \text { (lower is better } \\
\text { calibration) }\end{array}$} & \multicolumn{2}{|c|}{$\begin{array}{l}\text { IDI ( }>0 \text { is better } \\
\text { discrimination) }\end{array}$} \\
\hline & & value & $95 \%$ CI & value & $95 \% \mathrm{CI}$ & value & $95 \% \mathrm{CI}$ \\
\hline 1 & Base model: age, gender, ethnicity, NIHSS & 0.83 & $0.74,0.90$ & 30.9 & $22.2,39.8$ & & \\
\hline 2 & Base model plus ICH CT findings* & 0.87 & $0.80,0.92$ & 26.3 & $18.6,35.0$ & & \\
\hline \multicolumn{2}{|c|}{ Change in AUC, $\hat{S}$, or IDI (model 2-model 1) } & 0.05 & $-0.002,0.11$ & -4.2 & $-11.5,2.3$ & 0.12 & $-0.005,0.26$ \\
\hline 3 & Base model plus GCS ${ }^{\dagger}$ & 0.85 & $0.77,0.91$ & 28.8 & $19.6,38.0$ & & \\
\hline 4 & Model 3 plus ICH CT findings* & 0.87 & $0.80,0.93$ & 26.6 & $17.7,35.5$ & & \\
\hline \multicolumn{2}{|c|}{ Change in AUC, $\hat{S}$, or IDI (model 4-model 3) } & 0.02 & $-0.02,0.08$ & -2.0 & $-9.7,3.4$ & 0.065 & $-0.03,0.21$ \\
\hline
\end{tabular}

* CT findings for ICH were hemorrhage volume in cc, intraventricular hemorrhage (yes/no), and infratentorial hemorrhage (yes/no).

${ }^{\dagger}$ Modeled as a quadratic term due to non-linear association with log-odds of mortality.

among ICH cases in both model 2 and model 4. Adding CT findings to the base model containing demographics and NIHSS (comparing model 2 to model 1) resulted in a slight but non-significant improvement in AUC and $\hat{S}$, though IDI was improved. However, if GCS was added to the base model as an additional assessment of the clinical exam, there was no significant improvement in AUC, $\hat{S}$, or IDI with the addition of CT findings (comparing model 4 to model 3). The changes in AUC and the IDI when restricting to ICH cases alone are shown in figure 2. 


\section{Discussion}

We found that excluding CT data for ICH cases had little overall impact on model performance when predicting 30-day mortality in either a population of mixed ICH and IS, or when restricting to ICH cases alone. CT findings appeared to add relatively little benefit to model discrimination ability (AUC and IDI) or calibration $(\hat{S})$ beyond that provided by demographics, clinical stroke severity (NIHSS and GCS), and stroke type (when considering both ICH and IS). These findings have implications for stroke outcome studies where it may not be practical to have each CT scan individually coded, such as multicenter quality improvement projects, studies based on administrative data, or large-scale populationbased studies with many events.

Our findings do not contradict the well-known association between mortality and CT findings such as hemorrhage volume or location in ICH [1]. Indeed, ICH volume and infratentorial location were associated with mortality in all models. Rather, these results suggest that depending on the particular research question at hand, it may not be necessary to go to the extra effort and expense of obtaining detailed CT data for ICH cases. For example, if the goal of a model is to determine which predictor variables have the strongest association with post-stroke mortality, then ICH CT findings should be included. However, if the goal is to account for stroke mortality risk while investigating the impact of other factors (such as gender or adherence to quality indicators) on stroke outcome, CT data may not be necessary. Cost of obtaining the CT data may vary substantially depending on the study setting (e.g. single center vs. multicenter or population-based) and our results may help investigators to assess the value of obtaining CT data in their own studies.

The lack of influence of CT findings even when restricting to ICH cases alone may seem surprising when considering that the majority of existing ICH predictive models include CT findings as predictors [1]. However, this finding is not unprecedented: the Essen ICH score is a validated ICH predictive model based on age, level of consciousness, and NIHSS that does not include imaging findings and yet has been shown to compare favorably to other models which do incorporate CT findings [23]. It appears that substantial information about mortality risk is contained in demographic variables and clinical examination findings, and therefore addition of CT findings adds relatively little to model performance. We cannot completely exclude the possibility of a type II error, particularly with the reduction in sample size when re- stricting to ICH cases alone. However, the point estimates of the change in AUC and $\hat{S}$ in table 3 suggest that the absolute incremental benefit of CT data on model performance is relatively small.

Our results differed slightly depending on whether NIHSS alone or NIHSS plus GCS were included as measures of clinical severity. When considering the model with NIHSS as the only clinical severity measure (model 1), we found that there was an improvement in IDI, but not AUC or $\hat{S}$ with addition of CT findings. While AUC and IDI are both measures of the model's ability to discriminate patients likely to die from those likely to survive, disparate results can occur due to the underlying characteristics of these measures. Both AUC and IDI can be viewed as the model sensitivity averaged across the range of probability thresholds (i.e. a probability value above which one might 'classify' a patient as more likely to die). However, AUC is weighted to give relatively more importance to sensitivity values at lower probability thresholds, whereas the IDI gives equal weight to all probability thresholds [20]. Because AUC gives more weight at low threshold values, the effect of the seemingly small loss of sensitivity at low threshold values (shown by the fact that the sensitivity curve with CT findings in figure $2 b$ is below the curve for the model without CT findings at low cutoff values) is given more importance when calculating AUC compared to IDI. Therefore, the disparate results for AUC and IDI when comparing model 2 to model 1 may be reflective of CT findings improving model discrimination at higher probability thresholds.

This study has several limitations. Our results may not apply to other populations, or when considering outcomes other than 30-day mortality. We do not have data available on long-term functional outcome, such as 90 day modified Rankin Scale, for this cohort. While BASIC has a higher proportion of Mexican Americans than many other stroke populations, the 30-day mortality and relative proportion of IS and ICH are similar to prior reports from other populations [24]. We focused on 30-day mortality given the powerful and consistent association with ICH CT findings that has been extensively reported in the literature [1]. We did not review brain imaging studies for IS cases; however, imaging findings are not felt to be as useful a predictor of outcome for IS as for ICH [25]. We also did not include data on comorbid illness such as coronary artery disease. Adding additional predictors may have altered the values of the AUC, $\hat{S}$, and IDI, but would be unlikely to change conclusions about the change in these parameters with or without CT findings. 


\section{Acknowledgements}

This study was funded by the NIH and the National Institute of Neurological Disorders and Stroke (R01 NS38916). Dr. Zahuranec is supported by NIH grant K23 AG038731 from the National Institute on Aging.

\section{Disclosure Statement}

The authors have no conflicts of interest to disclose.

\section{References}

$\checkmark 1$ Ariesen MJ, Algra A, van der Worp HB, Rinkel GJ: Applicability and relevance of models that predict short term outcome after intracerebral haemorrhage. J Neurol Neurosurg Psychiatry 2005;76:839-844.

$\checkmark 2$ Broderick JP, Brott TG, Duldner JE, Tomsick T, Huster G: Volume of intracerebral hemorrhage. A powerful and easy-to-use predictor of 30-day mortality. Stroke 1993;24:987-993.

$\checkmark 3$ Hemphill JC, 3rd, Bonovich DC, Besmertis L, Manley GT, Johnston SC: The ICH score: a simple, reliable grading scale for intracerebral hemorrhage. Stroke 2001;32:891-897.

$\checkmark 4$ Heuschmann PU, Wiedmann S, Wellwood I, Rudd A, Di Carlo A, Bejot Y, Ryglewicz D, Rastenyte D, Wolfe CD: Three-month stroke outcome: the European Registers of Stroke (EROS) investigators. Neurology 2011;76: 159-165.

5 Fonarow GC, Reeves MJ, Smith EE, Saver JL, Zhao X, Olson DW, Hernandez AF, Peterson ED, Schwamm LH: Characteristics, performance measures, and in-hospital outcomes of the first one million stroke and transient ischemic attack admissions in get with the guidelines-stroke. Circ Cardiovasc Qual Outcomes 2010;3:291-302.

-6 Guyomard V, Fulcher RA, Redmayne O, Metcalf AK, Potter JF, Myint PK: Effect of dysphasia and dysphagia on inpatient mortality and hospital length of stay: a database study. J Am Geriatr Soc 2009;57:2101-2106.

$\checkmark 7$ Luengo-Fernandez R, Gray AM, Rothwell PM: Population-based study of determinants of initial secondary care costs of acute stroke in the United Kingdom. Stroke 2006; 37:2579-2587.

$\checkmark 8$ Shepardson LB, Youngner SJ, Speroff T, Rosenthal GE: Increased risk of death in patients with do-not-resuscitate orders. Med Care 1999;37:727-737.

$\checkmark 9$ Vaartjes I, Reitsma JB, Berger-van Sijl M, Bots ML: Gender differences in mortality after hospital admission for stroke. Cerebrovasc Dis 2009;28:564-571.
10 Morgenstern LB, Smith MA, Lisabeth LD, Risser JM, Uchino K, Garcia N, Longwell PJ, McFarling DA, Akuwumi O, Al-Wabil A, AlSenani F, Brown DL, Moye LA: Excess stroke in Mexican Americans compared with nonHispanic Whites: the Brain Attack Surveillance in Corpus Christi Project. Am J Epidemiol 2004;160:376-383.

11 Al-Wabil A, Smith MA, Moye LA, Burgin WS, Morgenstern LB: Improving efficiency of stroke research: the Brain Attack Surveillance in Corpus Christi study. J Clin Epidemiol 2003;56:351-357.

12 Piriyawat P, Smajsova M, Smith MA, Pallegar S, Al-Wabil A, Garcia NM, Risser JM, Moye LA, Morgenstern LB: Comparison of active and passive surveillance for cerebrovascular disease: the Brain Attack Surveillance in Corpus Christi (BASIC) project. Am J Epidemiol 2002;156:1062-1069.

13 Williams LS, Yilmaz EY, Lopez-Yunez AM: Retrospective assessment of initial stroke severity with the NIH stroke scale. Stroke 2000;31:858-862.

14 Zahuranec DB, Brown DL, Lisabeth LD, Gonzales NR, Longwell PJ, Eden SV, Smith MA, Garcia NM, Morgenstern LB: Differences in intracerebral hemorrhage between Mexican Americans and non-Hispanic whites. Neurology 2006;66:30-34.

15 Zahuranec DB, Gonzales NR, Brown DL, Lisabeth LD, Longwell PJ, Eden SV, Smith MA, Garcia NM, Hoff JT, Morgenstern LB: Presentation of intracerebral haemorrhage in a community. J Neurol Neurosurg Psychiatry 2006;77:340-344.

-16 Kothari RU, Brott T, Broderick JP, Barsan WG, Sauerbeck LR, Zuccarello M, Khoury J: The ABCs of measuring intracerebral hemorrhage volumes. Stroke 1996;27:1304-1305.

$\checkmark 17$ Lisabeth LD, Risser JM, Brown DL, AlSenani F, Uchino K, Smith MA, Garcia N, Longwell PJ, McFarling DA, Al-Wabil A, Akuwumi O, Moye LA, Morgenstern LB: Stroke burden in Mexican Americans: the impact of mortality following stroke. Ann Epidemiol 2006;16:33-40.

-18 Hanley JA, McNeil BJ: The meaning and use of the area under a receiver operating characteristic (ROC) curve. Radiology 1982;143: 29-36.
19 Harrell FE, Jr, Lee KL, Mark DB: Multivariable prognostic models: issues in developing models, evaluating assumptions and adequacy, and measuring and reducing errors. Stat Med 1996;15:361-387.

20 Pencina MJ, D’Agostino RB, Sr, D’Agostino RB, Jr, Vasan RS: Evaluating the added predictive ability of a new marker: from area under the ROC curve to reclassification and beyond. Stat Med 2008;27:157-172; discussion 207-212.

21 Hosmer DW, Hosmer T, Le Cessie S, Lemeshow S: A comparison of goodness-of-fit tests for the logistic regression model. Stat Med 1997;16:965-980.

22 Davison AC, Hinkley DV, Canty AJ: Bootstrap methods and their application. Cambridge; New York, NY, USA, Cambridge University Press, 1997, pp 223-229.

23 Weimar C, Benemann J, Diener HC: Development and validation of the Essen intracerebral haemorrhage score. J Neurol Neurosurg Psychiatry 2006;77:601-605.

24 Roger VL, Go AS, Lloyd-Jones DM, Adams RJ, Berry JD, Brown TM, Carnethon MR, Dai S, de Simone G, Ford ES, Fox CS, Fullerton HJ, Gillespie C, Greenlund KJ, Hailpern SM, Heit JA, Ho PM, Howard VJ, Kissela BM, Kittner SJ, Lackland DT, Lichtman JH, Lisabeth LD, Makuc DM, Marcus GM, Marelli A, Matchar DB, McDermott MM, Meigs JB, Moy CS, Mozaffarian D, Mussolino ME, Nichol G, Paynter NP, Rosamond WD, Sorlie PD, Stafford RS, Turan TN, Turner MB, Wong ND, Wylie-Rosett J: Heart disease and stroke statistics - 2011 update: a report from the American Heart Association. Circulation 2011;123:e18-e209.

25 Johnston KC, Wagner DP, Wang XQ, Newman GC, Thijs V, Sen S, Warach S: Validation of an acute ischemic stroke model: does diffusion-weighted imaging lesion volume offer a clinically significant improvement in prediction of outcome? Stroke 2007;38: 1820-1825. 